\title{
A New Method for Calibration of Kappa Angle in 3D Line of Sight Tracking System
}

\author{
Lu Ning ${ }^{1}$, Zhang Guosheng ${ }^{2}$, Chi Jiannan ${ }^{1}$ \\ ${ }^{1}$ School of Automation \& Electrical Engineering, University of Science and Technology Beijing, Beijing, PRC \\ ${ }^{2}$ Ministry of Transport of the People's Republic of China, Key Laboratory of Operation Safety Technology on Transport Vehicle, Beijng, PRC
}

\section{Email address:}

ln222xxx@163.com (Lu Ning), gs.zhang@rioh.cn (Zhang Guosheng), ustbjnc@ustb.edu.cn (Chi Jiannan)

\section{To cite this article:}

Lu Ning, Zhang Guosheng, Chi Jiannan. A New Method for Calibration of Kappa Angle in 3D Line of Sight Tracking System. Science Innovation. Vol. 6, No. 4, 2018, pp. 182-189. doi: 10.11648/j.si.20180604.12

Received: June24, 2018; Accepted: July 12, 2018; Published: July 20, 2018

\begin{abstract}
At present, the general idea of the 3D line of sight (LoS) estimation is as follows. First, the direction of the 3D line of gaze (LoG) can be reconstructed according to the visual characteristics of the eyeballs (pupil center coordinates, purkinje images coordinates, etc.). Then the transformation matrix of the direction of LoG and the direction of LoS can be calculated based on the Kappa angle between LoG and LoS. LoS can be estimated with known LoG. The transformation matrix between LoG and LoS is usually determined by the user calibration process of the gaze tracking system. Because the eyeball structure is unique and the Kappa is a space angle, the transformation matrix changes with the space location of the eyeball. This paper elaborates on the Kappa angle calibration problem in the gaze tracking system. A new calibration and calculation method of Kappa angle is proposed in this paper, which can solve the calculation of space Kappa angle in the case of head translation, pitch and rotation. Simulation and experimental results verify the effectiveness of the proposed method.
\end{abstract}

Keywords: Gaze Tracking, Kappa Angle, Line of Sight, Line of Gaze

\section{三维视线追踪系统中Kappa角标定问题研究}

卢宁 $^{1}$, 张国胜 ${ }^{2}$, 迟健男 ${ }^{1}$

${ }^{1}$ 自动化学院, 北京科技大学, 北京, 中国

2运输车辆运行安全技术交通行业重点实验室，交通运输部，北京，中国

\section{邮箱}

ln222xxx@163.com(卢宁), gs.zhang@rioh.cn(张国胜), ustbjne@ustb.edu.cn(迟健男)

\begin{abstract}
摘要：目前三维视线估计的一般思路是：首先根据眼球的可视化特征（瞳孔中心坐标、普尔钦斑坐标等）重建眼球的 光轴的方向, 再根据眼球光轴和视轴之间的Kappa角, 计算视轴与光轴之间的转换矩阵, 继而由转换矩阵将光轴换算 成眼球的视轴的方向。而眼球光轴和视轴之间的转换矩阵一般是通过视线追踪系统的用户标定过程来确定的。但是由 于眼球独特的结构和Kappa是一个空间角，因此仅仅通过用户标定所确定的眼球在标定位置处的转换矩阵是无法确定 眼球在非标定位置出的转换矩阵。本文对视线追踪系统中的Kappa角标定问题进行了深入的阐述，提出了一种新的 Kappa角标定和计算方法, 可以解决头部平移、俯仰和转动情况下的空间Kappa角的解算, 仿真和实验结果验证了本文 方法的有效性。
\end{abstract}

关键词: 视线追踪, Kappa角, 光轴, 视轴 


\section{1. 引言}

一般来说，基于视觉的视线追踪技术可以分为两类: 基于二维映射模型的视线估计方法 [1-5]和基于三维模型 的视线估计方法 [6-9]。二维视线检测系统通常由一个相机 和一个光源组成, 其中, 视线方向可以由用户标定时的映 射函数得到。但是, 这种系统对头部运动有很大的限制。 二维映射模型通常是用一个二阶多项式来表示注视点处 的睹孔中心与普尔钦斑的矢量差。而这些多项式系数大多 是特定的, 在用户标定过程中, 用户需要注视屏幕上的多 个标定点来标定用户的特定参数, 标定过程较为复杂。由 于二维插值法使用映射函数来表示人眼视线参数和屏幕 注视点之间的关系, 对于这种系统, 可以在不知道相机、 光源和屏幕之间的关系的情况下进行注视点估计。通过比 较发现, 三维视线追踪系统通常由多个相机和多个光源组 成。真实的三维视线可以通过相机坐标系下的人眼参数和 人眼成像模型来估计得到。文献[10]表明, 当人眼参数未 知时, 需要使用至少两个相机或至少两个点光源来用于计 算角膜曲率中心的位置。基于三维模型的方法, 通常是通 过使用光源坐标或普尔钦斑坐标来对眼球进行建模, 从而 估计人眼的一些固定参数, 如角膜曲率半径等。通过这些 参数, 可以利用眼球结构和成像模型来重建光轴, 继而通 过视轴与光轴之间的kappa角, 计算视轴与光轴的转换关 系, 从而计算将重构出的光轴转化为视轴。当视轴矢量和 系统坐标系下观察平面的位置确定后, 注视点即为视线与 观察面的交点。由于三维视线追踪系统可以实时估计视线 的空间位置, 因此这种系统可以在自然头部运动下工作, 而且能够简化的用户标定过程[11]。

眼球的结构如图1所示, 眼球视轴定义为通过视网膜 小凹和角膜曲率中心直线, 但是, 无法通过眼球的可视特 征直接估计视轴的方向。因此, 三维视线估计模型的一般 思路[12-13]是：首先，在用户标定过程中, 确定眼球的 Kappa角; 然后, 根据眼球的可视特征, 估计眼球光轴的 方向; 最后, 根据检测到的光轴方向和标定的Kappa角, 将眼球光轴的方向换算成眼球视轴的方向。可见, 对于三 维视线估计系统, kappa角的标定是用户标定中重要的、 不可回避的内容, 但目前很少有文献详细研究Kappa角的 标定问题, 根据现有文献检索, 目前三维视线估计的Kappa 角的标定问题没有得到有效的解决, 这也是目前的视线追 踪系统无法实现全自由头动下视线估计的根本原因。

Kappa角的标定主要有两种方法:

（1）标定位置处的空间Kappa角的测量方法 [14]。在 这种方法中, 用户盯视屏幕上的一个标定点, 这时可以根 据图像上眼球的可视特征, 如瞳孔中心、瞳孔边缘点和普 尔钦斑, 计算出此时的角膜曲率中心和眼球光轴方向。由 于标定点的位置坐标在相机坐标系中是已知的, 因此此时 的眼球视轴也是已知的。这样根据眼球在标定位置处的光 轴和视轴, 就可以计算出眼球光轴和视轴之间的Kappa角。 但是Kappa角是一个空间角, 对于任意位置的光轴, 即使
知道Kappa角的值, 也无法根据光轴和Kappa角来唯一的确 定视轴, 因此这种方法相当于没有真正地标定出实用的 kappa角。

（2）多标定点的平均Kappa角测量方法 [15]。这种方 法实际是标定眼球光轴和视轴之间的转换矩阵 (设为未知 的 $3 * 3$ 的矩阵）, 即眼睛盯视屏幕上多个标定点, 在盯视 每个标定点时, 计算此时眼球的光轴和视轴, 这样此时的 光轴向量乘以转换矩阵等于视轴向量, 可以构成包含九个 未知数的三个方程。同理, 当用户盯视 $\mathrm{n}$ 个标定点时, 就 可以构成 $3 \mathrm{n}$ 个方程, 只要方程的个数 $3 \mathrm{n}$ 大于 9 , 就可以构 成包含 9 个未知数的方程组, 采用最小二乘法等优化方法 即可求解出光轴和视轴之间的转换矩阵。显然这种方法仍 然无法求得真正的空间Kappa角或其矩阵, 当标定点足够 多, 并且在头部处于空间各个位置时盯视标定点才能求得 比较准确的眼球光轴和视轴之间的转换矩阵, 但这种用户 标定过程过于复杂，使三维视线估计失去了其实用的价值。

本文在深入探讨眼球Kappa角标定问题的本质和局限 性的基础上, 提出了一种新的Kappa角标定方法, 可以实 现在头部平移、俯仰和转动情况下, 准确地解算出空间 Kappa角的值, 实现由眼球光轴方向到视轴方向之间的转 换计算。仿真实验验证了本文方法的有效性, 鉴于Kappa 角标定在视线估计中的重要性, 本文是唯一专门对Kappa 角标定问题进行研究的文献。本文的其余的内容安排如下: 第二节阐述了眼球结构的特点, 详细分析了Kappa角标定 问题的实质和局限性。第三节中给出一种新的Kappa角标 定方法。第四节的仿真实验验证了本文方法的有效性。最 后在第五节中对全文进行了总结。

\section{2. 问题陈述}

\section{1. 人眼结构}

图1展示了视线追踪系统中所用的人眼结构。眼球是 一个近似的球形体, 眼睛的外层, 依次可见巩膜 (眼球中 的白色部分）、虹膜（眼球中圆形的黑色部分）和瞳孔。 角膜是一层透明的球形的保护膜, 覆盖在虹膜外面。虹膜 中心有一个环状的通孔, 称作曈孔, 它的作用是通过改变 孔径的大小来调节进入眼睛的光量。虹膜后面是晶状体, 是眼球中的一种透明的双面凸体, 在角膜和晶状体之间是 水样体, 在晶状体和视网膜之间是玻璃体。光线在进入眼 睛后穿过这一系列的光介质并在各个层面上经反射和折 射后才最后到达视网膜。视网膜位于眼球的后面, 它蕴含 大量感光细胞。而黄斑中央凹陷处的视网膜最薄, 只有色 素上皮细胞和视锥细胞两层细胞, 双极细胞和节细胞均斜 向周围排列。此处视锥细胞与双极细胞均一对一联系, 故 它是视网膜上视觉最敏锐的部位, 因此视网膜小凹可视为 人眼视觉中心。 


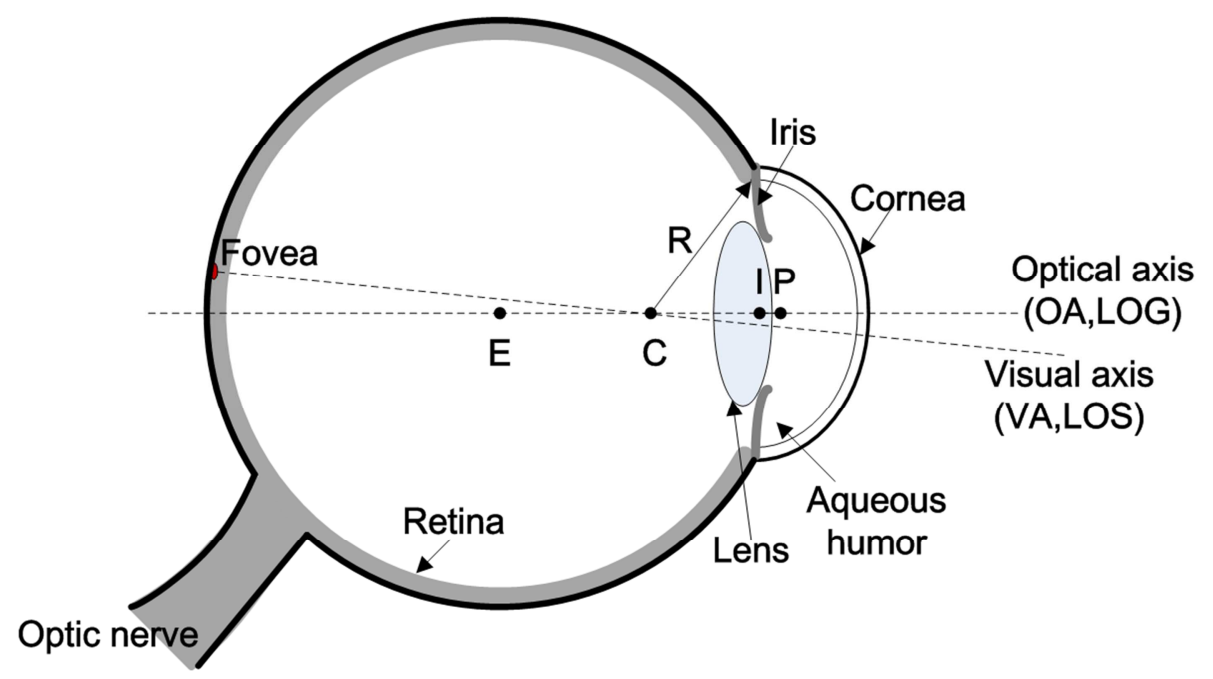

图1 人眼结构图。

光轴是眼球中心, 角膜曲率中心和瞳孔中心三点的连 线, 而连接黄斑中心和角膜曲率中心的连线成为视轴。它 们相交于角膜曲率中心, 其夹角通常称为Kappa角。通常, 视轴被认为是真实的注视方向, 因此视轴的计算是三维视 线估计的关键。但由于黄斑中心是无法从外部观察到的, 即不是可视特征, 所以很难直接用角膜曲率中心来计算视 线。而现有的三维视线估计模型中大多是先估计空间中人 眼的光轴, 然后通过视轴与光轴之间的角度偏移来获得视 轴。

由于眼球中心 $\mathrm{E}$, 角膜曲率中心 $\mathrm{C}$, 瞳孔中心P和虹膜 中心I都在光轴上, 其中P和I是相机所拍人眼图像中的可见 部分, $C$ 是光轴和视轴的交点, 因此, 这三点通常用于重 建光轴。由于光轴总是垂直于由瞳孔边缘或虹膜边缘所决 定的平面, 因此可以用瞳孔边缘或虹膜边缘所在平面的法 线近似表示。

\section{2. 眼球空间状态的表征及Kappa角计算问题}

假设头和眼球自由运动,即头部自由移动和转动,眼球 围绕眼球中心自由转动, 这样头部和眼球的运动的叠加构 成了眼球的三维自由运动。由上述眼球的结构特点可知, 在三维视线估计过程中, 眼球的光轴 $\mathrm{OA}$ 是可以通过眼球 的可视特征进行重建的。由于眼球是球形的, 瞳孔和虹膜 是以光轴为对称的圆形目标, 角膜是覆盖在虹膜上的球形 面, 角膜的曲率中心位于光轴上。因此, 如图2所示, 拍 摄眼睛图像, 从眼球的可视化特征 [16]（瞳孔、虹膜、亮 斑）, 以及由可视化特征推导出的计算特征（空间的角膜 曲率中心坐标、空间的瞳孔中心坐标) 都位于眼球光轴上, 无任何眼球光轴以外的特征可以利用来表述眼球的姿态, 因此对眼球空间状态的描述缺乏足够的信息。即头部运动 和眼球运动的叠加下眼球的空间运动如果包含绕眼球光 轴的转动的分量, 那么这个转动分量是无法通过眼球的特 征（可视特征和计算特征）来表征的。既使在配置红外光 源的视线追踪系统中, 光源在角膜上形成了反射亮斑, 由 于角膜近似球形, 则亮斑的位置仍然无法反映眼球绕其光 轴转动的信息。

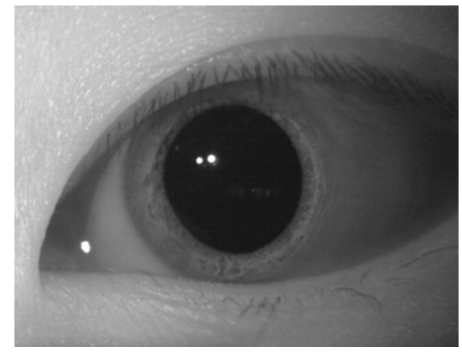

图2 人眼图像。

而 $\mathrm{kappa}$ 角是眼球光轴和视轴之间的空间角, 在确 定了眼球光轴的空间位置时, 如果无法确定眼球空间状 态的准确转换, 特别是无法确定眼球绕其光轴的转动量, 则就无法确定在眼球任何空间位置时的空间Kappa角。 因此在三维视线追踪系统中, 既使在每个时刻都能检测 出眼球光轴的方位, 也无法由光轴的方位推算出眼球视 轴的方位。这也是目前的视线追踪系统 (无论是二维系 统还是三维系统) 无法做到在全自由头动下视线方向估 计的原因。具体证明如下:

如图3所示, $C_{0}$ 为眼球的用户标定位置, 由眼球的结 构可知, 眼球中除了其光轴和光轴上的点可以用来表征眼 球空间状态的特征点, 再没有其它的可以由可视特征来计 算的特征点或线用来建立眼球坐标系。因此以眼球的角膜 曲率中心为坐标系原点 $\mathrm{O}$, 眼球的光轴为 $\mathrm{Z}$ 轴, 以与 $\mathrm{Z}$ 轴垂 直的平面内的任意两个两条互相垂直的直线作为 $\mathrm{X}, \mathrm{Y}$ 轴 来建立眼球坐标系。假设在用户标定过程中, 已经标定出 了眼球在标定位置处的眼球光轴和视轴之间的Kappa角。 在视线估计过程中, 眼球从用户标定的位置 $C_{0}$ 变化到另 一个空间位置 $C_{1}$, 其位置和姿态的改变, 可以用坐标系 的旋转和平移来表征。眼球从标定位置 $C_{0}$ 变化到任意空 间位置 $C_{1}$ 的过程, 就是眼球坐标系 $O-X Y Z$ 平移和旋转到 坐标系 $O^{\prime}-X^{\prime} Y^{\prime} Z^{\prime}$ 的过程。

假设在系统相机坐标系下, 眼球在标定位置 $C_{0}$ 处其 光轴方向向量为: 


$$
O_{0}=\left[\begin{array}{lll}
x_{0} & y_{0} & z_{0}
\end{array}\right]^{T}
$$

角膜曲率中心为:

$$
C_{0}=\left[\begin{array}{lll}
c_{x 0} & c_{y 0} & c_{z 0}
\end{array}\right]
$$

在任意位置时眼球光轴向量为:

$$
O_{1}=\left[\begin{array}{lll}
x_{1} & y_{1} & z_{1}
\end{array}\right]^{T}
$$

角膜曲率中心为:

$$
C_{1}=\left[\begin{array}{lll}
c_{x 1} & c_{y 1} & c_{z 1}
\end{array}\right]
$$

当眼球坐标系从位置 $C_{0}$ 旋转和平移到任意位置 $C_{1}$ 时, 其平移量为:

$$
T=\left[\begin{array}{lll}
c_{x 1}-c_{x 0} & c_{y 1}-c_{y 0} & c_{z 1}-c_{z 0}
\end{array}\right]^{T}
$$

其旋转矩阵 $\mathrm{R}$ 可以设为具有 3 个自由度的正交旋转矩 阵, 则:

$$
R=(I+S)(I-S)^{-1}
$$

其中,

$$
S=\left[\begin{array}{ccc}
0 & -a & -b \\
a & 0 & -c \\
b & c & 0
\end{array}\right]
$$

而 $\mathrm{a}, \mathrm{b}, \mathrm{c}$, 为三个独立的未知数。则标定位置 $C_{0}$ 的 眼球光轴与任意位置的眼球光轴的之间的变换矩阵即为 两个位置处眼球坐标系之间的变换矩阵:

$$
\left[\begin{array}{ll}
R & T \\
0 & 1
\end{array}\right] O_{0}=O_{1}
$$

由式(8)可得:

$$
\left[\begin{array}{ccc}
0 & -z_{0}-z_{1} & -y_{0}-y_{1} \\
-z_{0}-z_{1} & 0 & x_{0}+x_{1} \\
y_{0}+y_{1} & x_{0}+x_{1} & 0
\end{array}\right]\left[\begin{array}{l}
c \\
b \\
a
\end{array}\right]=\left[\begin{array}{l}
x_{1}-x_{0} \\
y_{1}-y_{0} \\
z_{1}-z_{0}
\end{array}\right]
$$

由于式(9)中参数矩阵的秩为 2 , 根据线性方程组的求 解特性可知, 无法求出 $\mathrm{a}, \mathrm{b}, \mathrm{c}$ 三个独立变量的唯一解。 此时旋转矩阵R无解。

\section{Kappa角标定方法}

Kappa角是眼球光轴与视轴的夹角。本文的kappa角标 定包含两个部分：（1）kappa角标定：在用户标定过程中, 标定出kappa角的值。（2）kappa角解算：在视线估计过 程中, 根据标定的kappa角, 实时计算当时的空间Kappa 角的值。
如图2所示, 在用户标定过程中, 以角膜曲率中心 $\mathrm{C}$ 为原点, 以眼球光轴为 $\mathrm{Z}$ 轴, 以与 $\mathrm{Z}$ 轴垂直并相互垂直的任 意两条直线分别为 $X$ 轴和 $Y$ 轴, 设立眼球坐标系。使用者 盯视屏幕上的标定点 $\mathrm{PC}$, 首先构建出眼球光轴, 而角膜曲 率中心和标定点坐标可以得到视轴, 这样就可以确定空间 Kappa角的值。

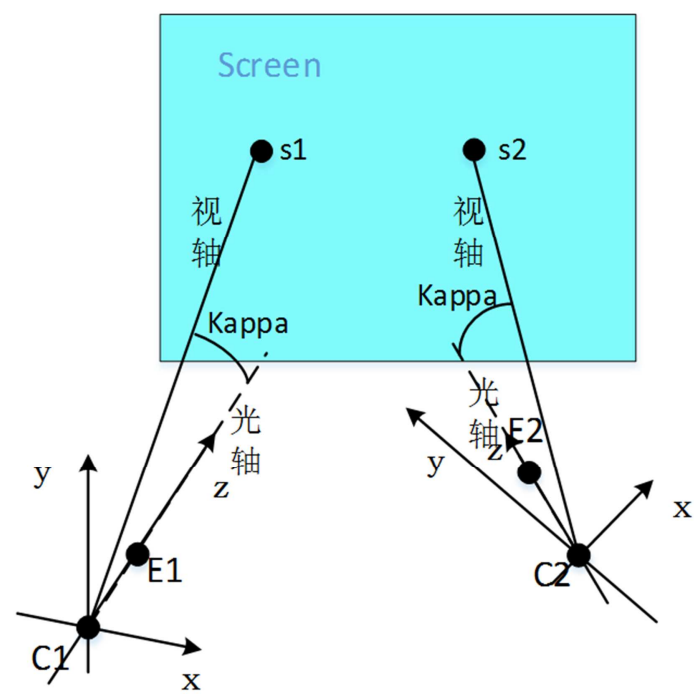

图3 Kappa角示意图。

$$
\cos \text { Kappa }=\frac{(E-C) \cdot\left(P_{c}-C\right)}{|E-C|\left|P_{c}-C\right|}
$$

在系统相机坐标系下, 眼球从标定位置旋转和平移到 任意位置时, 眼球坐标系原点平移量为:

$$
\left[\begin{array}{ccc}
c_{x 1}-c_{x 0} & c_{y 1}-c_{y 0} & c_{z 1}-c_{z 0}
\end{array}\right]^{\mathbf{T}}
$$

平移后, 眼球坐标系绕 $\mathrm{x}$ 轴旋转 $\alpha$ 角, 绕 $\mathrm{y}$ 轴旋转 $\beta$ 角, 绕 $\mathrm{z}$ 轴无转动。则标定位置的眼球光轴与任意位置的眼球 光轴的之间的变换矩阵即为两个位置处眼球坐标系之间 的变换矩阵:

$$
M O_{0}=O_{1}
$$

即:

$$
\left[\begin{array}{cccc}
\cos \beta & 0 & -\sin \beta & c_{x 1}-c_{x 0} \\
-\sin \alpha \sin \beta & \cos \alpha & -\sin \alpha \cos \beta & c_{y 1}-c_{y 0} \\
\cos \alpha \sin \beta & \sin \alpha & \cos \alpha \cos \beta & c_{z 1}-c_{z 0} \\
0 & 0 & 0 & 1
\end{array}\right]\left[\begin{array}{c}
x_{0} \\
y_{0} \\
z_{0} \\
1
\end{array}\right]=\left[\begin{array}{c}
x_{1} \\
y_{1} \\
z_{1} \\
1
\end{array}\right]
$$

由式(13)可得:

$$
\begin{gathered}
\left\{\begin{array}{c}
x_{0} \cos \beta-z_{0} \sin \beta=x_{1} \\
\cos ^{2} \beta+\sin ^{2} \beta=1
\end{array}\right. \\
\left\{\begin{array}{c}
-x_{0} \sin \alpha \sin \beta+y_{0} \cos \alpha-z_{0} \sin \alpha \cos \beta=y_{1} \\
x_{0} \cos \alpha \sin \beta+y_{0} \sin \alpha+z_{0} \cos \alpha \sin \beta=z_{1}
\end{array}\right.
\end{gathered}
$$


由方程组(14)可以求得 $\beta$ 角，由 $\beta$ 角和方程组(15)可 以求得 $\alpha$ 角。上述方程组可以得到两组解，根据双眼视线 汇聚于同一点的原则, 可以确定唯一的 $\alpha$ 角和 $\beta$ 角。在视 线估计过程中, 眼球在空间任意位置处重建眼球光轴后, 根据式(14)和(15)实时计算出眼球坐标系的旋转角 $\alpha$ 和 $\beta$, 再根据眼球的平移量:

$$
\left[\begin{array}{ccc}
c_{x 1}-c_{x 0} & c_{y 1}-c_{y 0} & c_{z 1}-c_{z 0}
\end{array}\right]^{\mathbf{T}}
$$

得到眼球坐标系的变换矩阵 $\mathrm{M}$ 。由于在眼球坐标系中, 眼球光轴和视轴之间具有确定的位置关系, 因此眼球在标 定位置处的视轴和在任意位置处的视轴之间的转换矩阵 也是 $M$, 则有:

$$
\mathbf{V}_{1}=\mathbf{M V}_{0}
$$

这样, 就可以由眼球在标定位置处的视轴方向计算出 眼球处于任意位置处的视轴方向上述Kappa角标定方法可 以解决头部平移、转动和俯仰情况下的Kappa角计算问题。

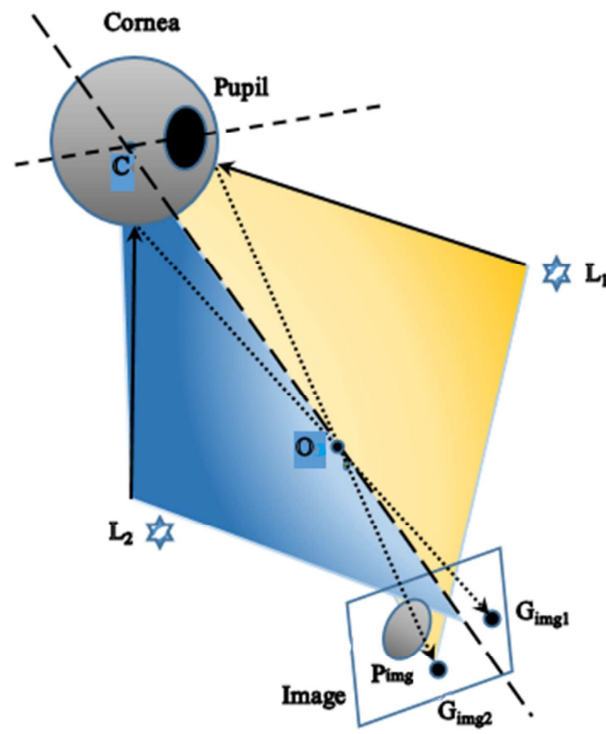

(a)

\section{4. 仿真实验}

\section{1. 仿真环境}

本文在Rhinoceros5.0环境下建立眼球仿真模型，并模 拟眼球在空间的运动, 提取眼球的结构的参数, 采用 Matlab- R2015a进行算法仿真运算, 如图7所示, 在相机坐 标系下, 建立眼球结构模型, 包括眼球光轴、眼球视轴、 眼球中心和角膜曲率中心等, 并以角膜曲率中心为原点。 根据建立的眼球结构模型, 建立眼球坐标系。得到眼球光 轴和眼球视轴在标定位置处的初始值. 然后模拟眼球在标 定位置处移动到空间任意位置, 记录每个空间位置处的光 轴的方向向量和角膜曲率中心的位置坐标。

\section{2. 角膜曲率中心计算}

由文献[1][5]可知, 角膜被看作是以 $\mathrm{C}$ 为球心, $r_{c}$ 为球 面的半径。点光源发出的光线照射到角膜上, 形成反射亮 斑, 亮斑在相机中成像。由反射定律可知: (1) 入射角 与反射角相等; (2) 点光源、入射光线、反射光线及法 线共面。

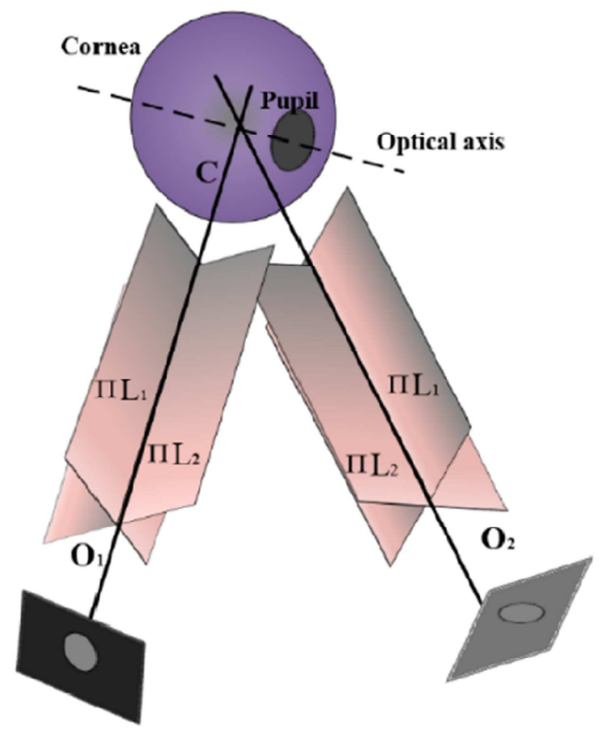

(b)

图4 求取角膜曲率中心原理图。

如图4（b）所示，设光源 L、入射光线、反射光线和 图像中亮斑 $G_{i m g}$ 构成的平面为 $\Pi_{L}$ 。角膜曲率中心 $\mathrm{C}$ 在角 膜球面上入射点的法线上, 因此, $\mathrm{C}$ 在平面 $\Pi_{L}$ 上, 如果 有两个点光源 L1和 L2, 在角膜表面发生两次反射, 则形成 两个普尔钦斑, 在相机像平面产生两个亮斑图像, 分别记 作 $G_{1 i m g}$ 和 $G_{2 i m g}$ 。每个光源和它产生的图像上的亮斑可确 定一个反射平面, 该平面包含角膜曲率中心 $\mathrm{C}$ 和相机光心 $\mathrm{O}$ 。

$$
\Pi_{\mathrm{L} 1}=L_{1} \times G_{\text {limg }}, \Pi_{\mathrm{L} 2}=L_{2} \times G_{2 \mathrm{img}}
$$

角膜曲率中心 $\mathrm{C}$ 在两个平面的交线上。同理，在双相 机系统中, 可以得到两条经过角膜曲率中心 $\mathrm{C}$ 的直线, 这 两条直线的交点即角膜曲率中心 $\mathrm{C}$ 。

\section{3. 光轴和视轴估计}

如图6所示, 本文注意到角膜曲率中心和空间中的瞳 孔边缘点的连线, 构成一个空间圆雉体 $\Omega$, 而这个圆锥 体的中心轴线就是眼球的光轴。本文方法通过两个相机 的图像上瞳孔的边缘点, 求取圆雉 $\Omega$ 上的多个连接角膜 曲率中心和瞳孔边缘点的直线, 则通过这些直线就能够 构造空间的圆雉体 $\Omega$, 再求得这个圆雉体的中心轴线, 就确定了眼球的光轴CE。两个点光源在角膜表面发生反 射形成两个普尔钦斑, 如图2所示, 相机 $O_{1}$ 拍摄得到的 
图像上的两个亮斑可分别表示为 $G_{1 i m g}$ 和 $G_{2 i m g}$, 利用反 射定律, 可以在相机 $\mathrm{O}_{2}$ 拍摄的图像上分别找到 $G_{1 i m g}$ 和 $G_{2 i m g}$ 的匹配点。如图5所示, 在相机 $\mathrm{O}_{1}$ 的图像上, 以 $G_{\text {limg }}$ 为原点, $G_{\text {limg }} G_{2 i m g}$ 所在的直线为x轴, 建立坐标系。
在相机 $\mathrm{O}_{2}$ 的图像上, 以 $G_{1 i m g}^{\prime}$ 为原点, $G_{1 i m g}^{\prime} G_{2 i m g}^{\prime}$ 所在 直线为 $\mathrm{x}$ 轴, 这样建立的坐标系就是统一的两个像平面的 坐标系。

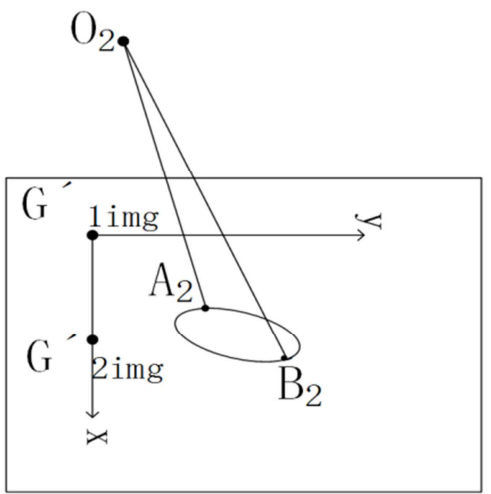

图5 匹配点示意图。

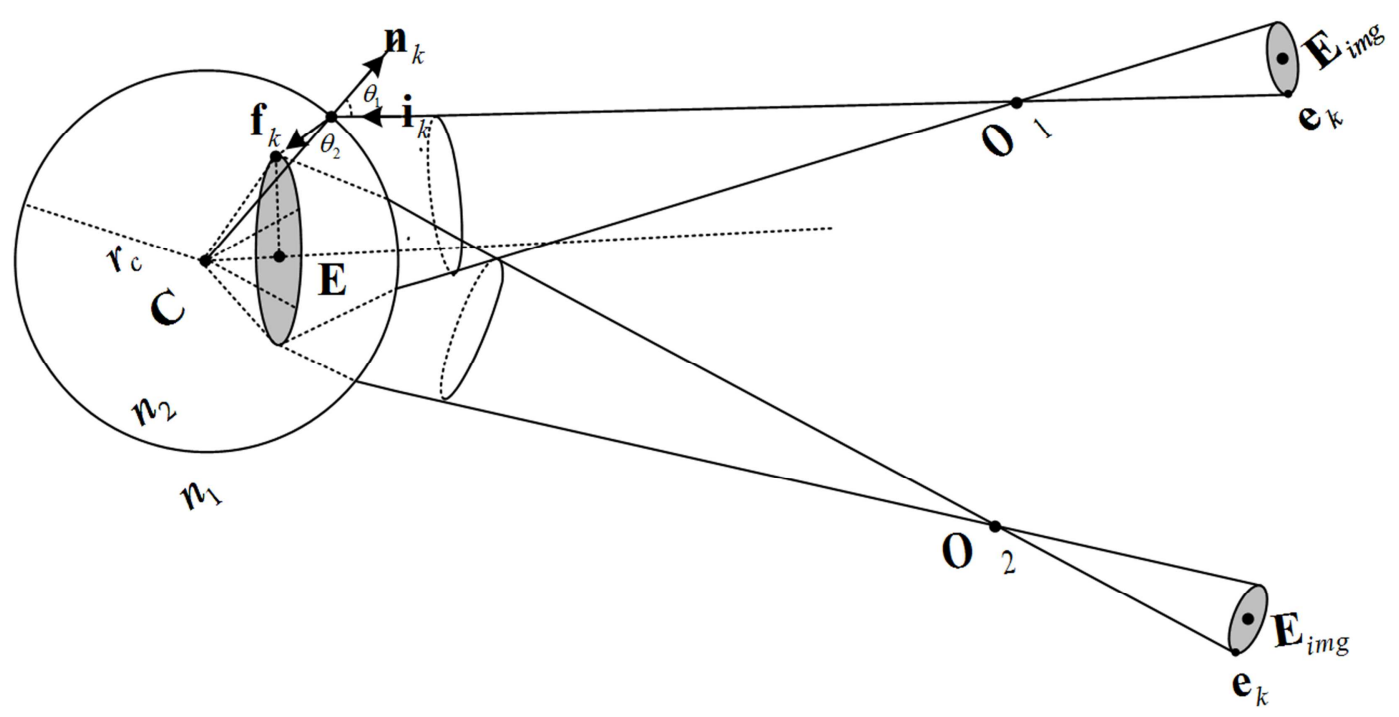

图6 眼球光轴重建示意图。

基于顺序约束法进行图像上瞳孔边缘点的匹配，如图 5 所示, 假设相机 $\mathrm{O}_{1}$ 图像上像点 $A_{1}$ 处于瞳孔像的最左端, 相机 $\mathrm{O}_{2}$ 图像上与像点 $A_{1}$ 匹配的像点 $A_{2}$ 不在瞳孔像的最 左端, 则在 $A_{2}$ 的左端存在瞳孔的像点 $\mathrm{M}$, 在相机 $\mathrm{O}_{1}$ 图像 上与像点 $\mathrm{M}$ 匹配的像点应该在 $A_{1}$ 的左端, 而事实上, $A_{1}$ 是 最左端的边缘像点, 不存在其他的像点与 $\mathrm{M}$ 匹配, 不符合 顺序约束。因此, 在相机 $\mathrm{O}_{2}$ 图像上, 与 $A_{1}$ 点匹配的像点 $A_{2}$ 一定处于瞳孔像的最左端。假设 $A_{1} 、 A_{2}$ 是瞳孔上 $A_{o}$ 点 的两个虚像点, $B_{1} 、 B_{2}$ 是瞳孔上 $B_{o}$ 点的两个虚像点,则 $A_{o} B_{o}$ 为瞳孔直径。根据上述顺序约束方法, 根据由普尔 钦斑所确定的两个统一的坐标系, 可以确定四对匹配点, 再根据这四对匹配点, 我们可以得到 $C_{4}^{2}=6$ 个统一的坐标 系, 在这六个统一的坐标系中, 再根据上述的顺序约束方 法, 共可以在双相机的睲孔边缘图像中确定 24 对匹配点。
由折射定律可知，入射光线、折射光线、法线在同一 平面上。已知角膜曲率中心 $\mathrm{C}$, 两个相机的光心 $o_{1} 、 o_{2}$, 图像瞳孔边缘点 $A_{1} 、 A_{2} 、 B_{1} 、 B_{2}$ 。C 在眼球光轴上, $A_{o}$ 在入射光线上, $o_{1} 、 A_{1}$ 在折射光线上, 因此, C、 $A_{o} 、 o_{1}$ 、 $A_{1}$ 共面。同理可得 $\mathrm{C} 、 A_{o} 、 o_{2} 、 A_{2}$ 共面。两个平面分别 记作 $\Pi_{A 1} 、 \Pi_{A 2}$, 可表示为:

$$
\begin{aligned}
& \Pi_{A 1}=C o_{1} \times o_{1} A_{1} \\
& \Pi_{A 2}=C o_{2} \times o_{2} A_{2}
\end{aligned}
$$

平面 $\Pi_{A 1} 、 \Pi_{A 2}$ 的交线为 $C A_{o}$ 。同理, C、 $B_{o} 、 o_{1}$ 、 $B_{1}$ 共面, C、 $B_{o} 、 o_{2} 、 B_{2}$ 共面, 两个平面分别记作 $\_$B 1 、 П_B2，可表示为:

$$
\Pi_{B 1}=C o_{1} \times o_{1} B_{1}
$$




$$
\Pi_{B 2}=\mathrm{Co}_{2} \times \mathrm{O}_{2} \mathrm{~B}_{2}
$$

平面 $\Pi_{B 1} 、 \Pi_{B 2}$ 的交线为 $C B_{o}$ 。这样, 对于两个相机 图像中所有的瞳孔边缘匹配点 $D_{1} 、 E_{1} 、 F_{1} \ldots$ 和 $D_{2} 、 E_{2}$ 、 $F_{2} \ldots$, 都可以求得相应的空间直线 $C D_{o} 、 C E_{o} 、 C F_{o} \ldots$ 。 则 $C A_{o} 、 C B_{o} 、 C D_{o} 、 C E_{o} 、 C F_{o} \ldots$ 等直线构成以 $\mathrm{C}$ 点为 顶点的空间圆雉体, 而这个圆雉体的中轴线即为光轴, 其 方向向量为:

$$
L o G=\frac{C A_{o}}{\left|C A_{o}\right|}+\frac{C B_{o}}{\left|C B_{o}\right|}+\frac{C D_{o}}{\left|C D_{o}\right|}+\frac{C E_{o}}{\left|C E_{o}\right|}+\frac{C F_{o}}{\left|C F_{o}\right|} \ldots
$$

根据构建的光轴和标定的Kappa角, 可以实时计算出 $3 \mathrm{D}$ 视线方向:

$$
L o S=M \cdot L o G
$$

\section{4. 仿真结果}

实验过程中, 首先记录人眼的初始位置信息, 包括角 膜中心的坐标, 光轴方向向量和视轴方向向量。接下来在 绕任意两个轴依次旋转不同的角度, 如本实验中就依次围 绕X轴和 $Y$ 轴分别旋转不同的角度。记录每次旋转后的角 膜中心坐标和光轴坐标。

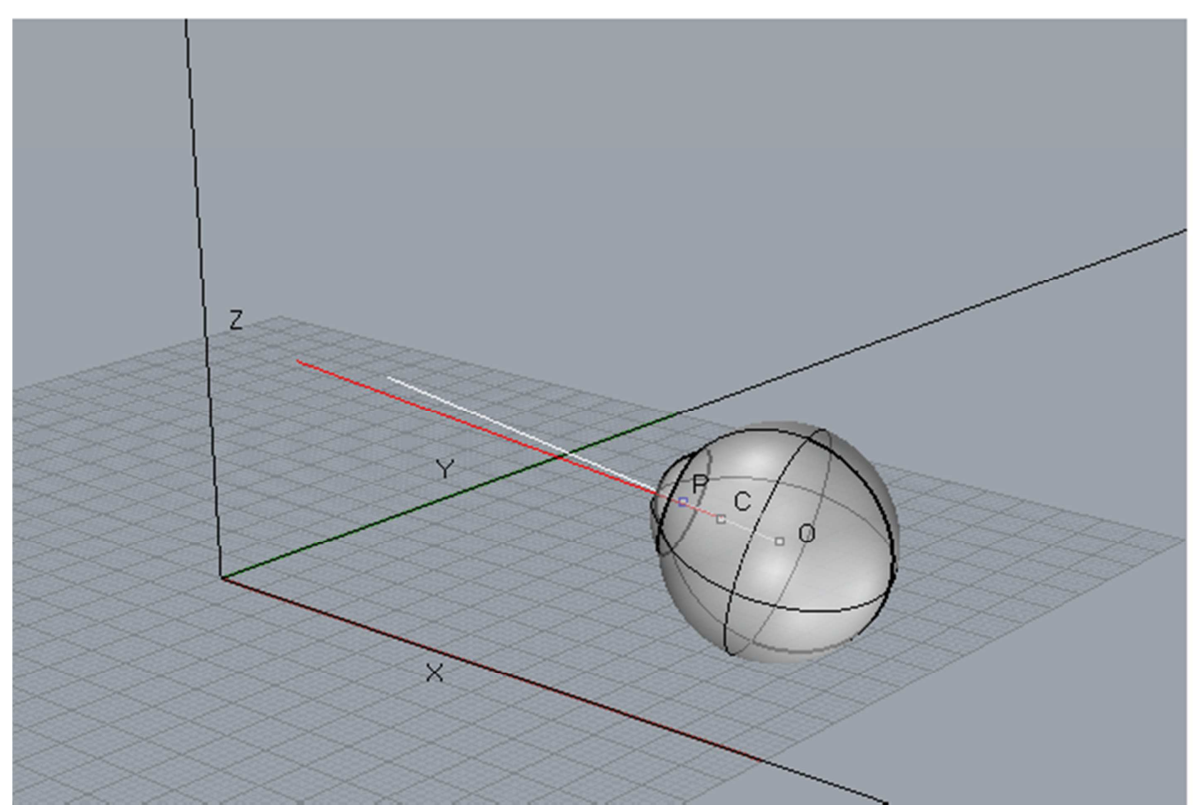

图7 仿真模型示意图 (红色线为视轴, 白色线为光轴)。

根据4.2和4.3节的方法计算眼球坐标系的由标定位置到空间任意位置的坐标系转换矩阵, 最后根据眼球坐标系的转 换矩阵，计算眼球在空间某个位置处的眼球光轴的方向向量。仿真结果如图8所示。

\section{仿真误差}

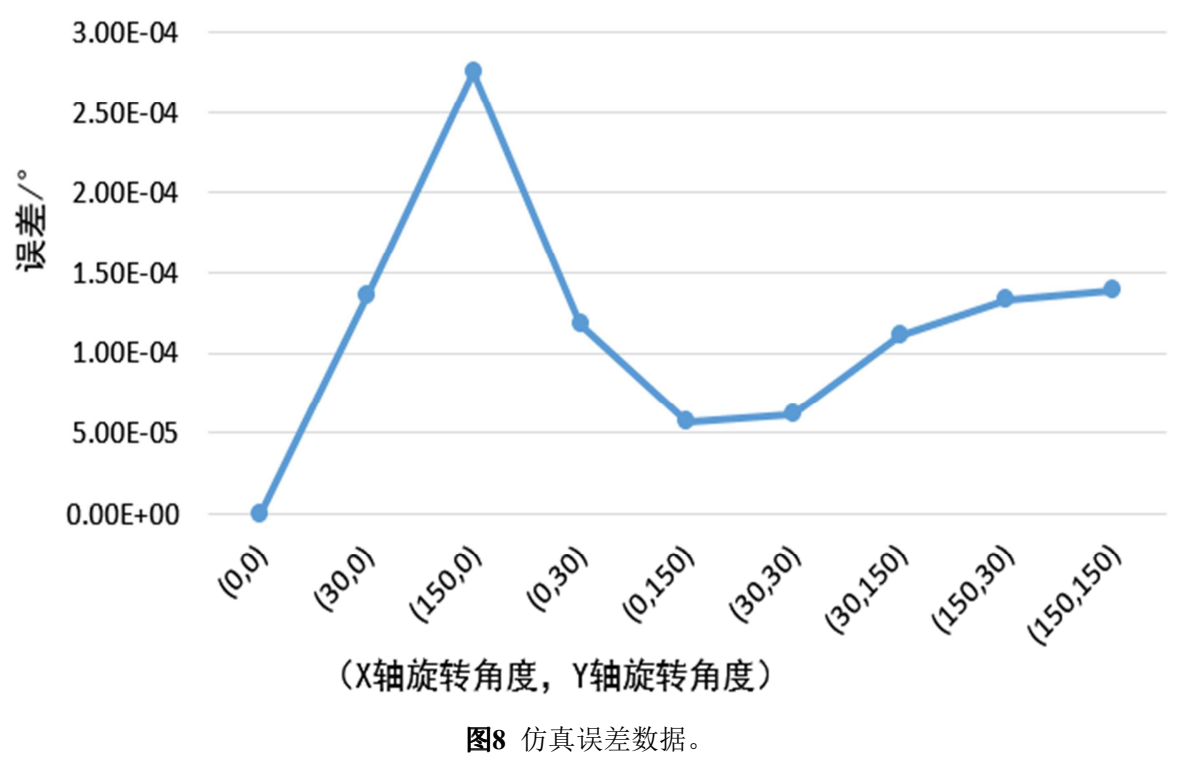


由仿真数据可知, 发现眼球在任意空间位置处的视 轴单位向量的求解的值与真实值的误差很小, 属于软件 计算的误差范围, 因此仿真结果表明本文的算法是可行 的。

\section{5.结论}

目前三维视线估计的一般思路是: 首先根据眼球的可 视化特征 (睲孔中心坐标、普尔钦斑坐标等) 重建眼球的 光轴的方向, 再根据眼球光轴和视轴之间的Kappa角, 换 算成眼球的视轴的方向。而眼球光轴和视轴之间的Kappa 角一般是通过视线追踪系统的用户标定过程来确定的。但 是由于眼球独特的结构和Kappa是一个空间角，因此仅仅 通过用户标定所确定的眼球在标定位置处的kappa角的值 是无法确定眼球在非标定位置出的空间Kappa角的值。本 文对视线追踪系统中的Kappa角标定问题进行了深入的阐 述, 提出了一种新的Kappa角标定和计算方法, 可以解决 头部平移、俯仰和转动情况下的空间Kappa角的解算, 仿 真和实验结果验证了本文方法的有效性。

\section{致谢}

本文为北京市自然科学基金（4172040）和交通运输 车辆运行安全技术重点实验室开放项目的阶段性成果之 一。

\section{参考文献}

[1] Chamberlain L. Eye Tracking Methodology; Theory and Practice [J]. Qualitative Market Research, 2013 (2).

[2] Choi K A, Ma C, Ko S J. Improving the usability of remote eye gaze tracking forhuman-device interaction [J]. Consumer Electronics, IEEE Transactions on, 2014, 60(3): 493-498.

[3] Zhu J, Yang J. Subpixel Eye Gaze Tracking[C].IEEE International Conference on Automatic Face and Gesture Recognition, 2002. Proceedings. 2002:124-129.

[4] Morimoto C H, Mimica M R M. Eye gaze tracking techniques for interactive applications $[\mathrm{J}]$. Computer Vision \& Image Understanding, 2005, 98(1):4-24.

[5] Jose Sigut and Sid-Ahmed Sidha. Iris Center Corneal Reflection Method for Gaze TrackingUsing Visible Light[J]. IEEE TRANSACTIONS ON BIOMEDICAL ENGINEERING, 2011,58(2):411-421.
[6] Shao G, Che M, Zhang B, et al. A Novel Simple 2D Model of Eye Gaze Estimation[C]. International Conference on Intelligent Human-Machine Systems \& Cybernetics. IEEE Computer Society, 2010:300-304.

[7] Valenti R, Sebe N, Gevers T. Combining Head Pose and Eye Location Information for Gaze Estimation [J]. IEEE Transactions on Image Processing, 2012, 21(2):802-815.

[8] Shih S W, Liu J. A novel approach to 3-D gaze tracking using stereo cameras.[J]. IEEE Transactions on Systems Man \& Cybernetics Part B Cybernetics A Publication of the IEEE Systems Man \& Cybernetics Society, 2004, 34(1):234-45.

[9] Hennessey C, Noureddin B, Lawrence P. A single camera eye-gaze tracking system with free head motion [J]. Proceedings of Etra Eye Tracking Research \& Applications Symposium, 2006:87-94.

[10] Elias Daniel G, Moshe E. General theory of remote gaze estimation using the pupil center and corneal reflections [J]. IEEE Transactions on Biomedical Engineering, 2006, 53(6):1124-1133.

[11] Dong H Y, Chung M J. Eye-mouse under large head movement for human-computer interface[C]. IEEE International Conference on Robotics and Automation, 2004. Proceedings. ICRA. IEEE, 2004:237-242 Vol.1.

[12] ED Guestrin, M Eizenman. Remote point-of-gaze estimation with free head movements requiring a single-point calibration. International Conference of the IEEE Engineering in Medicine \& Biology Society, 2007, 2007:4556-60.

[13] Sheng-Wen Shih, Jin Liu. A Novel Approach to 3D Gaze Tracking Using Stereo Cameras[J]. IEEE TRANSZETIONS ON SYSTEMS MAN AND CYBERNETICS-PART B: CYBERNETICS.2004, 34(1):234-256.

[14] Arantxa Villanueva and Rafael Cabeza. A Novel Gaze Estimation System With One Calibration Point[J]. IEEE TRANSACTIONS ON SYSTEMS, MAN, AND CYBERNETICS-PART B: CYBERNETICS, VOL. 38, NO. 4, AUGUST 2008.

[15] Zhu Z, Ji Q. Robust real-time eye detection and tracking under variable lighting conditions and various face orientations $[\mathrm{J}]$.Computer Vision and Image Understanding, 2005, 98(1): 124-154.

[16] Chih-Chuan Lai, Sheng-Wen Shih, Member, IEEE, and Yi-Ping Hung. Hybrid Method for 3-D Gaze Tracking Using Glint and Contour Features[J]. IEEE TRANSACTIONS ON CIRCUITS AND SYSTEMS FOR VIDEO TECHNOLOGY, VOL. 25, NO. 1, JANUARY 2015. 